\title{
ON COMPATIBILITY CONDITIONS AND MANILOLDS OF SOLUTIONS TO ONE CLASS OF OVERDETERMINED SYSTEMS OF SECOND ORDER PARTIAL DIFFERENTIAL EQUATIONS
}

\author{
R. PIROV
}

\begin{abstract}
In the work we consider a class of overdetermined system of second order partial differential equations for one unknown function involving one or two second order derivatives in the right hand side. We find the compatibility conditions and prove theorems on existence and uniqueness of solutions involving at most six arbitrary constants.
\end{abstract}

Keywords: overdetermined systems, compatbility conditions, manifolds of solutions, cross differentiation.

Mathematics Subject Classification: 35N10

\section{INTRODUCTION AND FORMULATION OF THE PROBLEM}

In monograph [1], systems of first order partial differential equations with one unknown function were considered. In [2] and [3], there were studied some second order quasilinear system of equations for one unknown function. These studies were continued in works [4]-6].

In the present work we consider nonlinear systems of four second order differential equations, where the unknown function depends on three independent variables and the right hand sides involve nonlinearly one or two of the derivatives $U_{x x}, U_{y y}, U_{z z}, U_{x y}, U_{y z}, U_{x z}$.

We restrict ourselves by considering one system in each group, namely:

$$
U_{x x}, U_{x y}, U_{x z}, U_{y z}=f^{i}\left(x, y, z, U, U_{x}, U_{y}, U_{z}, U_{y y}\right), \quad i=\overline{1,4}
$$

and

$$
U_{x x}, U_{x y}, U_{x z}, U_{y z}=f^{i}\left(x, y, z, U, U_{x}, U_{y}, U_{z}, U_{y y}, U_{z z}\right), \quad i=\overline{1,4} .
$$

Using the same notation $f^{i}$ in systems (1.1) and (1.2) for the functions depending on various amount of the arguments ( 8 and 9 , respectively), is justified by the fact that these systems are studied independently. In these systems $U=U(x, y, z)$ is the unknown function which is sought in class $C^{4}\left(\Pi_{0}\right)$; here

$$
\Pi_{0}=\left\{(x, y, z):\left|x-x_{0}\right| \leqslant a,\left|y-y_{0}\right| \leqslant a,\left|z-z_{0}\right| \leqslant a\right\}
$$

for some $a>0$.

R. Pirov, On COMPatibility CONDitions and manilolds of SOlutions to one Class of OVerdeTERMINED SYSTEMS OF SECOND ORDER PARTIAL DIFFERENTIAL EQUATIONS.

(c) PIROV R. 2016.

Submitted December 3, 2015. 
The main method of studying the above mentioned systems is replacing the first and second order derivatives by new functions, passing to systems with more variables and establishing connection with quite well studied total differential systems (TDS) (see, for instance, [2]).

\section{STUDY OF SYSTEM (1.1)}

We consider first system (1.1). By $\Pi=\Pi(a, b)$ we denote a rectangle in space $\mathbb{R}^{8}$ introduced by the inequalities:

$$
\begin{array}{llll}
\left|x-x_{0}\right| \leqslant a, & \left|y-y_{0}\right| \leqslant a, & \left|z-z_{0}\right| \leqslant a, & \left|U-U_{0}\right| \leqslant b, \\
\left|U_{x}-U_{x}^{0}\right| \leqslant b, & \left|U_{y}-U_{y}^{0}\right| \leqslant b, & \left|U_{z}-U_{z}^{0}\right| \leqslant b, & \left|U_{y y}-U_{y y}^{0}\right| \leqslant b .
\end{array}
$$

Superscript " 0 " stands for the value of a function at point $\left(x_{0}, y_{0}, z_{0}\right)$. Let $f^{i} \in C^{2}(\Pi), i=\overline{1,4}$.

We make the change $U_{x}=p(x, y, z), U_{y}=q(x, y, z), U_{z}=W(x, y, z), U_{y y}=q_{y}=\tau(x, y, z)$. Then we have obvious identities: $p_{y} \equiv q_{x}, q_{z} \equiv W_{y}$ and $p_{z} \equiv W_{x}$. Under these changes system (1.1) becomes:

$$
\begin{aligned}
& U_{x}=P(x, y, z), \quad U_{y}=q(x, y, z), \quad U_{z}=W(x, y, z) \\
& p_{x}, p_{y}, p_{z}=f^{i}(x, y, z ; U, p, g, W, \tau), \quad i=\overline{1,3} \\
& q_{x}=f^{2}(x, y, z ; U, p, g, W, \tau), \quad q_{y}=\tau, \quad q_{z}=f^{4}(x, y, z ; U, p, g, W, \tau), \\
& W_{x}=f^{3}(x, y, z ; U, p, g, W, \tau), \quad W_{y}=f^{4}(x, y, z ; U, p, g, W, \tau) .
\end{aligned}
$$

The identities for the mixed derivatives $p_{y z}=p_{z y}, q_{y z}=q_{z y}, q_{x y}=q_{y x}$ and $q_{x z}=q_{z x}$ by simple transformations lead us to the equations:

$$
\begin{aligned}
f_{W}^{2} W_{z}+f_{\tau}^{2} \tau_{z}-f_{\tau}^{3} \tau_{y}= & f_{y}^{3}-f_{z}^{2}+f_{U}^{3} q-f_{U}^{2} W \\
& +f_{p}^{3} f^{2}-f_{P}^{2} f^{3}-f_{q}^{3} \tau-f_{q}^{2} f^{4}+f_{W}^{3} f^{4}\left(\equiv L^{1}\right), \\
-f_{\tau}^{4} \tau_{y}+\tau_{z}=f_{y}^{4}+f_{U}^{4} q+ & f_{p}^{4} f^{2}+f_{q}^{4} \tau+f_{W}^{4} f^{4}\left(\equiv L^{2}\right) \\
\tau_{x}-f_{\tau}^{2} \tau_{y}=f_{y}^{2}+f_{U}^{2} q+ & f_{p}^{2} f^{2}+f_{q}^{2} \tau+f_{W}^{2} f^{4}\left(\equiv L^{3}\right) \\
f_{W}^{2} W_{z}+f_{\tau}^{2} \tau_{z}-f_{\tau}^{4} \tau_{x}= & f_{x}^{4}-f_{z}^{2}+f_{U}^{4} p-f_{U}^{2} W+f_{p}^{4} f^{1} \\
& -f_{p}^{2} f^{3}+f_{q}^{4} f^{2}-f_{q}^{2} f^{4}+f_{W}^{4} f^{3}\left(\equiv L^{4}\right) .
\end{aligned}
$$

As $f_{W}^{2} \neq 0$ and $f_{\tau}^{2} f_{\tau}^{4}-f_{\tau}^{3} \neq 0$, by (2.2)-(2.5) we find algebraically $W_{z}, \tau_{y}, \tau_{x}$ and $\tau_{z}$ as

$$
W_{z}, \tau_{y}, \tau_{x}, \tau_{z}=f^{j}(x, y, z, U, p, q, W, \tau), \quad j=\overline{5,8}
$$

where

$$
\begin{aligned}
& f_{W}^{2} f^{5}=L^{4}-f_{\tau}^{2} L^{2}+f_{\tau}^{2} f^{3}, \\
& \left(f_{\tau}^{2} f_{\tau}^{4}-f_{\tau}^{3}\right) f^{6}=L^{1}-L^{4}-f_{\tau}^{4} f^{3}, \\
& f^{7}=L^{3}+f_{\tau} f^{2}, f^{8}=f_{\tau}^{4} f^{6}+L^{2} .
\end{aligned}
$$

Joining (2.6) and (2.1), we arrive at TDS w.r.t. five unknown functions. The identities for the mixed derivatives $p_{y z}=p_{z y}, q_{x y}=q_{y x}, q_{y z}=q_{z y}$ in systems (2.1) and (2.6) can be checked by simple calculations; they also used in completion of the system. Other nine identities $q_{z x}=q_{x z}$, $p_{x y}=p_{y x}, p_{z x}=p_{x z}, W_{x y}=W_{y x}, W_{y z}=W_{z y}, W_{x z}=W_{z x}, \tau_{x y}=\tau_{y x}, \tau_{y z}=\tau_{z y}$ and $\tau_{x z}=\tau_{z x}$ 
are finally transformed to the following functional relations:

$$
\begin{aligned}
H^{1}(x, y, z ; U, p, q, W, \tau) \equiv & f_{z}^{2}+f_{U}^{2} W+f_{p}^{2} f^{3}+f_{q}^{2} f^{4}+f_{W}^{2} f^{5}+f_{\tau}^{2} f^{8} \\
& -f_{x}^{4}-f_{U}^{4} p-f_{p}^{4} f^{1}-f_{q}^{4} f^{2}-f_{W}^{4} f^{3}-f_{\tau}^{4} f^{7}=0, \\
H^{2}(x, y, z ; U, p, q, W, \tau)= & f_{y}^{1}+f_{U}^{1} q+f_{p}^{1} f^{2}+f_{q}^{1} \tau+f_{W}^{1} f^{4}+f_{\tau}^{1} f^{6} \\
& -f_{x}^{2}-f_{U}^{2} p-f_{p}^{2} f_{1}-f_{q}^{2} f^{2}-f_{\tau}^{2} f^{7}=0 \\
H^{3}(x, y, z ; U, p, q, W, \tau) \equiv & f_{z}^{1}+f_{U}^{1} W+f_{p}^{1} f^{3}+f_{q}^{1} f^{4}+f_{W}^{1} f^{5}+f_{\tau}^{1} f^{8} \\
& -f_{x}^{3}-f_{U}^{3} p-f_{p}^{3} f^{1}-f_{q}^{3} f^{2}-f_{W}^{3} f^{3}-f_{\tau}^{3} f^{7}=0, \\
H^{4}(x, y, z ; U, p, q, W, \tau) \equiv & f_{y}^{3}+f_{U}^{3} q+f_{p}^{3} f^{2}+f_{q}^{3} \tau+f_{W}^{3} f^{4}+f_{\tau}^{3} f^{6} \\
& -f_{x}^{4}-f_{U}^{4} p-f_{p}^{4} f^{1}-f_{q}^{4} f^{2}-f_{W}^{4} f^{3}-f_{\tau}^{4} f^{7}=0, \\
H^{5}(x, y, z ; U, p, q, W, \tau) \equiv & f_{z}^{4}+f_{U}^{4} W+f_{p}^{4} f^{3}+f_{q}^{4} f^{4}+f_{W}^{4} f^{5} \\
& -f_{y}^{5}-f_{U}^{5} f^{2}-f_{p}^{5} f^{2}-f_{q}^{5} \tau-f_{W}^{5} f^{4}-f_{\tau}^{5} f^{6}=0, \\
H^{6}(x, y, z ; U, p, q, W, \tau) \equiv & f_{z}^{3}+f_{U}^{3} W+f_{p}^{3} f^{3}+f_{q}^{3} f^{4}+f_{W}^{3} f^{5} \\
& -f_{x}^{5}-f_{U}^{5} f^{1}-f_{p}^{5} f^{1}-f_{q}^{5} f^{2}-f_{W}^{5} f^{3}-f_{\tau}^{5} f^{7}=0, \\
H^{7}(x, y, z ; U, p, q, W, \tau) \equiv & f_{y}^{7}+f_{U}^{7} q+f_{p}^{7} f^{2}+f_{q}^{7} \tau+f_{W}^{7} f^{4}+f_{\tau}^{7} f^{6} \\
& -f_{x}^{6}-f_{U}^{6} p-f_{p}^{6} f^{1}-f_{q}^{6} f^{2}-f_{W}^{6} f^{3}-f_{\tau}^{6} f^{7}=0, \\
H^{8}(x, y, z ; U, p, q, W, \tau) \equiv & f_{z}^{6}+f_{U}^{6} W+f_{p}^{6} f^{3}+f_{q}^{6} f^{4}+f_{w}^{6} f^{5}+f_{\tau}^{6} f^{8} \\
& -f_{y}^{8}-f_{U}^{8} q-f_{p}^{8} f^{2}-f_{q}^{8} \tau-f_{W}^{8} f^{4}-f_{\tau}^{8} f^{6}=0, \\
H^{9}(x, y, z ; U, p, q, W, \tau) \equiv & f_{z}^{7}+f_{U}^{7} W+f_{p}^{7} f^{3}+f_{q}^{7} f^{4}+f_{W}^{7} f^{5}+f_{\tau}^{7} f^{8} \\
& -f_{x}^{8}-f_{U}^{8} f^{1}-f_{p}^{8} f^{1}-f_{q}^{8} f^{2}-f_{W}^{8} f^{3}-f_{\tau}^{8} f^{7}=0 .
\end{aligned}
$$

Since (2.1) and (2.6) are obtained by equivalent transformations from (1.1), the initial conditions for this system are defined by the formulae

$$
[U]_{0}=c_{1}, \quad\left[U_{x}\right]_{0}=c_{2}, \quad\left[U_{y}\right]_{0}=c_{3}, \quad\left[U_{z}\right]_{0}=c_{4}, \quad\left[U_{y y}\right]_{0}=c_{5} .
$$

On the basis of the above described scheme of the study transforming a nonlinear system to a quasilinear total differential system, the next theorem is proved.

Theorem 2.1. Let $f^{i} \in C^{2}(\Pi), f_{W}^{2} \neq 0$ and $f_{\tau}^{2} f_{\tau}^{4}-f_{\tau}^{3} \neq 0$. If all nine conditions (2.8) and $\alpha<\min (a, b / M), M=\max \left|f^{i}\right|$ hold true identically w.r.t. $U, U_{x}, U_{y}, U_{z}, U_{y y}$, then problem (1.1), (2.9) in $\Pi(\alpha, b)$ is uniquely solvable in $C^{4}\left(\Pi_{0}\right)$.

In other words, under the conditions of Theorem 2.1, the manifold of solutions to system (1.1) contains five arbitrary constants $c_{1}, c_{2}, c_{3}, c_{4}$ and $c_{5}$.

Remark 2.1. Assume that one of conditions (2.8) does not hold identically and assume that it leads to the relation $\tau=\varphi\left(x, y, z, U, U_{x}, U_{y}, U_{z}\right), \varphi \in C^{1}(\Pi)$. Then the solution contains four arbitrary constants $c_{1}, \ldots, c_{4}$.

Let us provide two examples demonstrating Theorem 2.1 and Remark 2.1. As the first example we consider an overdetermined system: $U_{x x}=U_{y y}, U_{x y}=U_{z}, U_{x z}=-U_{y y}, U_{y z}=-U_{y y}$. After appropriate changes this system is reduced to TDS w.r.t. five unknowns $U, p, q, W$ and 
$\tau:$

$$
\left\{\begin{array}{lll}
U_{x}=p, & U_{y}=q, & U_{z}=W, \\
p_{x}=\tau, & p_{y}=W, & p_{z}=-\tau, \\
q_{x}=W, & q_{y}=\tau, & q_{z}=-\tau, \\
W_{x}=-\tau, & W_{y}=-\tau, & W_{z}=\tau, \\
\tau_{x}=-\tau, & \tau_{y}=-\tau, & \tau_{z}=\tau .
\end{array}\right.
$$

The assumptions of Theorem 2.1 hold true; in particular, relations (2.8) are satisfied identically. Therefore, the considered system is compatible. It is easy to see that its solution is the function $U(x, y, z)=c_{1} e^{-x-y+z}+c_{2}(x y+z)+c_{3} y+c_{4} x+c_{5}$.

As the second example, we consider the overdetermined system: $U_{x x}=U_{x}, U_{x y}=U_{z}$, $U_{x z}=-U_{y y}, U_{y z}=U_{y y}$. It is reduced to TDS

$$
\left\{\begin{array}{lll}
U_{x}=p, & U_{y}=q, & U_{z}=W, \\
p_{x}=p, & p_{y}=W, & p_{z}=-\tau, \\
q_{x}=W, & q_{y}=\tau, & q_{z}=\tau, \\
W_{x}=-\tau, & W_{y}=\tau, & W_{z}=\tau, \\
\tau_{x}=\tau, & \tau_{y}=\tau, & \tau_{z}=-\tau .
\end{array}\right.
$$

All relations (2.8) hold true identically except the relation corresponding to the identity $P_{x y}=$ $P_{y x}$. This relation leads us to the equation $\tau+W=0$. By Remark 2.1 this system is reduced to TDS for four unknown functions:

$$
\left\{\begin{array}{lll}
U_{x}=p, & U_{y}=q, & U_{z}=W, \\
p_{x}=p, & p_{y}=W, & p_{z}=W, \\
q_{x}=W, & q_{y}=-W, & q_{z}=-W \\
W_{x}=W, & W_{y}=-W, & W_{z}=-W .
\end{array}\right.
$$

Therefore, the considered system is compatible; it is easy to see that its solution is the function $U(x, y, z)=-c_{1} e^{x-y-z}+c_{2} y+c_{3} e^{x}+c_{4}$.

\section{STUdy OF SYSTEM (1.2)}

We proceed to nonlinear system (1.2). By $\Pi=\Pi(a, b)$ we denote a rectangle in space $\mathbb{R}^{9}$ defined by the inequalities: $\left|x-x_{0}\right| \leqslant a,\left|y-y_{0}\right| \leqslant a,\left|z-z_{0}\right| \leqslant a,\left|U-U_{0}\right| \leqslant b,\left|U_{x}-U_{x}^{0}\right| \leqslant b$, $\left|U_{y}-U_{y}^{0}\right| \leqslant b,\left|U_{z}-U_{z}^{0}\right| \leqslant b,\left|U_{y y}-U_{y y}^{0}\right| \leqslant b,\left|U_{z z}-U_{z z}^{0}\right| \leqslant b$. Let $f^{i} \in C^{2}(\Pi), i=\overline{1,4}$.

The change $U_{x}=p(x, y, z), U_{y}=q(x, y, z), U_{z}=W(x, y, z), U_{y y}=q_{y}=\tau(x, y, z)$ and $U_{z z}=W_{z}=\theta(x, y, z)$ transforms system (1.2) in the following quasilinear first order system

$$
\left\{\begin{array}{lll}
U_{x}=P, & U_{y}=q, & U_{z}=W, \\
P_{x}=f^{i}, & P_{y}=f^{2}, & P_{z}=f^{3}, \\
q_{x}=f^{2}, & q_{y}=\tau, & q_{z}=f^{4} \\
W_{x}=f^{3}, & W_{y}=f^{4}, & W_{z}=\theta .
\end{array}\right.
$$

We note that the made change ensures $p_{y}=q_{x}, q_{z}=W_{y}, p_{z}=W_{x}$ identically. The identities $p_{x y}=p_{y x}, q_{x y}=q_{y x}, W_{x y}=W_{y x}, p_{x z}=p_{z x}, q_{x z}=q_{z x}, W_{x z}=W_{z x}, p_{y z}=p_{z y}, q_{y z}=q_{z y}$, 
$W_{y z}=W_{z y}$ lead us to the following nine equations

$$
\begin{aligned}
&-f_{\tau}^{2} \tau_{x}+f_{\tau}^{1} \tau_{y}-f_{\theta}^{2} \theta_{x}+f_{\theta}^{1} \theta_{y}= f_{x}^{2}+f_{U}^{2} p+f_{p}^{2} f^{1}+f_{q}^{2} f^{2}+f_{W}^{2} f^{3} \\
&-f_{y}^{1}-f_{U}^{1} q-f_{p}^{1} q-f_{p}^{1} f^{2}-f_{q}^{1} \tau-f_{W}^{1} f^{4}, \\
& \tau_{x}-f_{\tau}^{2} \tau_{y}-f_{\theta}^{2} \theta_{y}=f_{y}^{2}+f_{U}^{2} q+f_{p}^{2} f^{2}+f_{q}^{2} \tau+f_{W}^{2} f^{4} \\
&-f_{\tau}^{4} \tau_{x}+f_{\tau}^{3} \tau_{y}-f_{\theta}^{4} \theta_{x}+f_{\theta}^{3} \theta_{y}= f_{y}^{4}+f_{U}^{4} p+f_{p}^{4} f^{1}+f_{q}^{4} f^{2}+f_{W}^{4} f^{3} \\
&-f_{y}^{3}-f_{U}^{3} q-f_{p}^{3} f^{2}-f_{W}^{3} f^{4}, \\
&-f_{\tau}^{3} \tau_{x}+f_{\tau}^{1} \tau_{z}-f_{\theta}^{3} \theta_{x}+f_{\theta}^{1} \theta_{z}= f_{x}^{3}+f_{U}^{3} p+f_{p}^{3} f^{1}+f_{q}^{3} f^{2}+f_{W}^{3} f^{3} \\
&-f_{z}^{1}-f_{U}^{1} W-f_{p}^{1} f^{3}-f_{q}^{1} f^{4}-f_{W}^{1} \theta, \\
&-f_{\tau}^{4} \tau_{x}+f_{\tau}^{2} \tau_{z}-f_{\theta}^{4} \theta_{x}+f_{\theta}^{2} \theta_{z}= f_{x}^{4}+f_{U}^{4} p+f_{p}^{4} f^{1}+f_{q}^{4} f^{2}+f_{W}^{4} f^{3} \\
&-f_{z}^{2}-f_{U}^{2} W-f_{p}^{2} f^{3}-f_{q}^{2} f^{4}-f_{W}^{2} \theta, \\
&-f_{\tau}^{3} \tau_{z}+\theta_{x}-f_{\theta}^{3} \theta_{z}=f_{z}^{3}+f_{U}^{3} W+f_{p}^{3} f^{3}+f_{q}^{3} f^{4}+f_{W}^{3} \theta \\
&-f_{\tau}^{3} \tau_{y}+f_{\tau}^{2} \tau_{z}-f_{\theta}^{3} \theta_{y}+f_{\theta}^{2} \theta_{z}=f_{y}^{3}+f_{U}^{3} q+f_{p}^{3} f^{2}+f_{W}^{3} f^{4}-f_{z}^{2} \\
&-f_{U}^{2} W-f_{p}^{2} f^{3}-f_{q}^{2} f^{4}-f_{W}^{2} \theta \\
&-f_{\tau}^{4} \tau_{y}+\tau_{z}-f_{\theta}^{4} \theta_{y}=f_{y}^{4}+f_{U}^{4} q+ f_{p}^{4} f^{2}+f_{q}^{4} \tau+f_{W}^{4} f^{4} \\
&-f_{\tau}^{4} \tau_{z}+\theta_{y}-f_{\theta}^{4} \theta_{z}=f_{z}^{4}+f_{U}^{4} W+f_{p}^{4} f^{3}+f_{q}^{4} f^{4}+f_{W}^{4} \theta
\end{aligned}
$$

Since in some neighbourhood of point $\left(x_{0}, y_{0}, z_{0} ; U^{0}, U_{x}^{0}, U_{y}^{0}, U_{z}^{0}, U_{y y}^{0}, U_{z z}^{0}\right)$ the rank of the $6 \times 9$ matrix

$$
\left\|\begin{array}{cccccc}
-f_{\tau}^{2} & f_{\tau}^{1} & 0 & f_{\theta}^{2} & f_{\theta}^{1} & 0 \\
1 & -f_{\tau}^{2} & 0 & 0 & -f_{\theta}^{2} & 0 \\
-f_{\tau}^{4} & f_{\tau}^{2} & 0 & -f_{\theta}^{4} & f_{\theta}^{3} & 0 \\
-f_{\tau}^{3} & 0 & f_{\tau}^{1} & -f_{\theta}^{3} & 0 & f_{\theta}^{1} \\
-f_{\tau}^{4} & 0 & f_{\tau}^{2} & -f_{\theta}^{4} & 0 & f_{\theta}^{2} \\
0 & 0 & -f_{\tau}^{3} & 1 & 0 & -f_{\theta}^{3} \\
0 & -f_{\tau}^{3} & f_{\tau}^{2} & 0 & -f_{\theta}^{3} & f_{\theta}^{2} \\
0 & -f_{\tau}^{4} & 1 & 0 & -f_{\theta}^{4} & 0 \\
0 & 0 & -f_{\tau}^{4} & 0 & 1 & -f_{\theta}^{4}
\end{array}\right\|
$$

formed by the coefficients at the derivatives $\tau_{x}, \tau_{y}, \tau_{z}, \theta_{x}, \theta_{y}, \theta_{z}$ is equal 6 (that can be checked by straightforward calculations), by (3.2) we find

$$
\tau_{x}, \tau_{y}, \tau_{z}, \theta_{x}, \theta_{y}, \theta_{z}=f^{k}(x, y, z ; U, p, q, W, \tau, \theta), \quad k=\overline{5,10} .
$$

We observe that functions $f^{k}, k=\overline{5,10}$, are expressed explicitly via functions $f^{1}, \ldots, f^{4}$, and their first partial derivatives. Joining (3.4) and (3.1), we arrive at TDS for six unknown functions $U, p, q, W, \tau, \theta$ :

$$
\left\{\begin{array}{lll}
U_{x}=P, & U_{y}=q, & U_{z}=W, \\
p_{x}=f^{1}, & p_{y}=f^{2}, & p_{z}=f^{3}, \\
q_{x}=f^{2}, & q_{y}=\tau, & q_{z}=f^{4}, \\
W_{x}=f^{3}, & W_{y}=f^{4}, & W_{z}=\theta, \\
\tau_{x}=f^{5}, & \tau_{y}=f^{6}, & \tau_{z}=f^{7}, \\
\theta_{x}=f^{8}, & \theta_{y}=f^{9}, & \theta_{z}=f^{10} .
\end{array}\right.
$$


In system (3.5) equivalent to (1.2) first 12 identities for mixed derivatives hold true immediately while other six $\tau_{x y}=\tau_{y x}, \tau_{y z}=\tau_{z y}, \tau_{x z}=\tau_{z x}, \theta_{x y}=\theta_{y x}, \theta_{y z}=\theta_{z y}, \theta_{x z}=\theta_{z x}$ after simple transformations lead to six functional equations

$$
\begin{aligned}
\widetilde{H}^{1}(x, y, z ; U, p, q, W, \tau, \theta) \equiv & f_{y}^{5}-f_{x}^{6}+f_{U}^{5} q-f_{U}^{6} p+f_{p}^{5} f^{2}-f_{p}^{6} f^{1}+f_{q}^{5} \tau-f_{q}^{6} f^{2} \\
& +f_{W}^{5} f^{4}-f_{W}^{6} f^{3}+f_{\theta}^{5} q-f_{\theta}^{6} f^{8}+f_{\tau}^{5} f^{6}-f_{\tau}^{6} f^{5}=0, \\
\widetilde{H}^{2}(x, y, z ; U, p, q, W, \tau, \theta) \equiv & f_{z}^{6}-f_{y}^{7}+f_{U}^{6} W-f_{U}^{7} f^{4}+f_{p}^{6} f^{3}-f_{p}^{7} f^{2}+f_{q}^{6} f^{4}-f_{q}^{7} \tau \\
& +f_{W}^{6} \theta-f_{W}^{7} f^{4}+f_{\tau}^{6} f^{7}-f_{\tau}^{7} f^{6}+f_{\theta}^{6} f^{10}-f_{\theta}^{7} f^{9}=0, \\
\widetilde{H}^{3}(x, y, z ; U, p, q, W, \tau, \theta) \equiv & f_{z}^{5}-f_{x}^{7}+f_{U}^{5} W-f_{U}^{7} p+f_{p}^{5} f^{3}-f_{p}^{7} f^{1}+f_{q}^{5} f^{4}-f_{q}^{7} f^{2} \\
& +f_{W}^{5} \theta-f_{W}^{7} f^{3}+f_{\tau}^{5} f^{7}-f_{\tau}^{7} f^{5}+f_{\theta}^{5} f^{10}+f_{\theta}^{7} f^{8}=0, \\
\widetilde{H}^{4}(x, y, z ; U, p, q, W, \tau, \theta) \equiv & f_{y}^{8}-f_{x}^{9}+f_{U}^{8} q-f_{U}^{9} p+f_{p}^{8} f^{2}-f_{p}^{9} f^{1}+f_{q}^{8} \tau-f_{q}^{9} f r^{2} \\
& +f_{W}^{8} f^{4}-f_{W}^{9} f^{3}+f_{\tau}^{8} f^{6}-f_{\tau}^{5}+f_{\theta}^{8} f^{9}-f_{\theta}^{9} f^{8}=0, \\
\widetilde{H}^{5}(x, y, z ; U, p, q, W, \tau, \theta) \equiv & f_{z}^{9}-f_{y}^{10}+f_{U}^{9} W-f_{U}^{10} q+f_{p}^{9} f^{3}-f_{p}^{10} f^{2}+f_{q}^{9} f^{4} \\
& -f_{q}^{10} \tau+f_{W}^{9} \theta-f_{W}^{10} f^{4}+f_{\tau}^{9} f^{7}-f_{\tau}^{10} f^{6}+f_{\theta}^{10} f^{9}=0, \\
\widetilde{H}^{6}(x, y, z ; U, p, q, W, \tau, \theta) \equiv & f_{z}^{8}-f_{x}^{10}+f_{U}^{8} W-f_{U}^{10} p+f_{p}^{8} f^{3}-f_{p}^{10} f^{1}+f_{q}^{8} f^{4}-f_{q}^{10} f^{2} \\
& +f_{W}^{8} \theta-f_{W}^{10} f^{3}+f_{\tau}^{8} f^{7}-f_{\tau}^{10} f^{5}+f_{\theta}^{8} f^{10}-f_{\theta}^{10} f^{8}=0 .
\end{aligned}
$$

It is clear that if in some neighbourhood of the point $\left(x_{0}, y_{0}, z_{0} ; U_{0}, p_{0}, q_{0}, W_{0}, \tau_{0}, \theta_{0}\right)$ the identities $H^{i}(x, y, z ; U, p, q, W, \tau, \theta) \equiv 0, i=\overline{1,6}$, hold true, then system (3.5) is completely integrable and we can apply total differential theory [7], [8] to the following problem with initial data:

$$
[U]_{0}=c_{1}, \quad[p]_{0}=c_{2}, \quad[q]_{0}=c_{3}, \quad[W]_{0}=c_{4}, \quad[\tau]_{0}=c_{5}, \quad[\theta]_{0}=c_{6},
$$

which in comparison with original system (1.2) is transformed to the problem

$$
[U]_{0}=c_{1}, \quad\left[U_{x}\right]_{0}=c_{2}, \quad\left[U_{y}\right]_{0}=c_{3}, \quad\left[U_{z}\right]_{0}=c_{4}, \quad\left[U_{x x}\right]_{0}=c_{5}, \quad\left[U_{z z}\right]_{0}=c_{6} .
$$

Theorem 3.1. Let $f^{i} \in C^{2}(\Pi)$ and in some neighbourhood of the point

$$
\left(x_{0}, y_{0}, z_{0} ; U^{0}, U_{x}^{0}, U_{y}^{0}, U_{z}^{0}, U_{x x}^{0}, U_{z z}^{0}\right)
$$

the identities $\widetilde{H}^{i} \equiv 0, i=\overline{1,6}$, hold true, where functions $\widetilde{H}^{i}$ are introduced by formulae (3.6)). Let $\alpha<\min (a, b / M), M=\max \left|f^{i}\right|$. Then problem (1.2), (3.7) in $\Pi(\alpha, b)$ is uniquely solvable in class $C^{4}\left(\Pi_{0}\right)$.

In particular, this theorem implies that under its assumptions, the manifold of the solutions to system (1.2) contains six arbitrary constants $c_{1}, \ldots, c_{6}$.

\section{BIBLIOGRAPHY}

1. E. Goursat. Leçons sur l'intégration des équations aux dérivées partielles du premier ordre. A. Hermann, Paris (1921).

2. L.G. Mikhailov. Some overdetermined systems of partial differential equations with two unknown functions. Donish, Dushanbe (1986). (in Russian).

3. A.V. Zhiber, R.D. Murtazina, I.T. Khabibullin, A.B. Shabat. Characteristic Lie rings and nonlinear integrable equations. Institute of Computer Studies, Moscow-Izhevsk (2012) (in Russian). 
4. R. Pirov. To theory of nonlinear overdetermined system of second order partial differential equations in a space // Izv. TO MAN VSh. 1, 85-90 (2010). (in Russian).

5. R. Pirov. On existence of harmonic solutions to one overdetermined system of four second order partial differential equations in a space // Vestn. Peduniver. Dushanbe. 2(38), 3-6 (2011).

6. R. Pirov. On compatibility of some overdetermined system of second order partial differential equations for two unknown functions in the plane // Dokl. AN Resp. Tadzhikistan. 54:5, 359-366 (2011).

7. Ph. Hartman. Ordinary differential equations. John Wiley \& Sons (1964).

8. V.V. Stepanov. Course of differential equations. Glavnoe izd-vo fiz.-matem. liter., Moscow (1958). (in Russian).

Pirov Rakhmon,

Tadzhik State Pedagogical University named after S. Aini, Rudaki av. 121,

734003, Dushanbe, Republic of Tadzhikistan

E-mail: pirov_60@mail.ru 